\title{
Current quests in nucleosynthesis: present and future neutron- induced reaction measurements
}

\author{
J. Praena ${ }^{1,2, a}$, M. Pignatari ${ }^{3}$, P.F. Mastinu ${ }^{4}$, G. Martín-Hernández ${ }^{5}$, G. Prete ${ }^{4}$, J.M. Quesada ${ }^{1}$, \\ and M. Sabaté-Gilarte ${ }^{1,2}$ \\ ${ }^{1}$ Universidad de Sevilla, Spain \\ ${ }^{2}$ Centro Nacional de Aceleradores (US-JA-CSIC), Seville, Spain \\ ${ }^{3}$ Department of Physics, University of Basel, Klingelbergstrasse 82, CH-4056 Basel, Switzerland \\ ${ }^{4}$ Laboratory Nazionali di Legnaro, INFN, Padova, Italy \\ ${ }^{5}$ Centro de Aplicaciones Tecnólogicas y Desarrollo Nuclear, $5^{\text {ta }}$ y 30, Playa, La Habana, Cuba
}

\begin{abstract}
We present some open questions in nucleosynthesis focused on the measurement of relevant neutron capture cross-sections and on new experimental methods. We review the recent ${ }^{63} \mathrm{Ni}(n, \gamma)$ experiment carried out at the $\mathrm{n} \_\mathrm{TOF}$ facility at CERN and its astrophysical implications as well as future experiments and opportunities at n_TOF. We argue some improvements in the measurement of cross-sections by activation arising from a new method for the generation of stellar neutron spectra. We show preliminary results of the experimental validation of the method. We discuss the astrophysical implications of the ${ }^{181} \mathrm{Ta}(n, \gamma)$ stellar cross-section measured with this method. Finally, we describe challenging experiments consisting of in situ radioactive ion beams and stellar neutron beams.
\end{abstract}

\section{Introduction.}

The work of $\mathrm{B}^{2} \mathrm{HF}$ stated that the stars are the seat of the origin of the elements, see [1] and references therein. They explained some features of the atomic abundance curve showing the mechanisms by which the elements heavier than hydrogen are produced in stellar environments and the mechanisms that could distribute the elements on a cosmic scale, as the ejection of the material by supernovae, novae, giant stages and planetary nebulae. $\mathrm{B}^{2} \mathrm{HF}$ proposed that the neutron-capture reactions and the subsequent $\beta$-decays are the responsible of the formation of the major part of the elements heavier than iron by different processes (slow or $s$, rapid or $r$ ) being about half of the element abundances due to the $s$-process while the other half to the $r$-process. The synthesis of the remaining part of isotopes, as the proton-rich, was explained mainly by the $p$-process, involving $(p, \gamma)$ and $(\gamma, n)$ reactions. The nuclear reactions ${ }^{13} \mathrm{C}(\alpha, n)$ and ${ }^{22} \mathrm{Ne}(\alpha, n)$ were proposed as neutron sources.

At present, the $s$-process distribution in the solar system distribution is usually divided in three components, see [2] and references therein: the weak $s$-process, responsible for most of the $s$-process abundances between $\mathrm{Fe}$ and $\mathrm{Sr}$, the main $s$-process component, producing most of the s-process elements between $\mathrm{Sr}$ and $\mathrm{Pb}$, and the strong s-process, responsible for half of the solar ${ }^{208} \mathrm{~Pb}$ and the

\footnotetext{
ae-mail: jpraena@us.es
} 
$s$-process abundance of $\mathrm{Bi}$. In particular, the weak $s$-process is activated in massive stars, mainly during the convective He-burning core and the following convective C-burning shell, while the main $s$-process and the strong $s$-process are made by low-mass stars during the Asymptotic Giant Branch phase (AGB) [3]. The origin of the $r$-process is less clear and several scenarios have been proposed, e.g., neutrino-driven winds associated to core-collapse supernovae and neutron stars mergers [4]. The $p$-process is mainly due to the activation of photodisintegration reactions $(\gamma, n),(\gamma, \alpha)$ and $(\gamma, p)$ in extreme explosive conditions in core-collapse supernovae [5] and eventually in thermonuclear supernovae [6]. Also the production of elements between $\mathrm{Sr}$ and $\mathrm{Ba}$ is still matter of debate in the scientific community. Indeed, the $s$-process contribution from both massive stars and AGB stars cannot account for the full $s$-process abundances of those elements observed in the solar system. As a consequence, a new unknown component called Lighter Element Primary Process has been proposed [7]. The origin of such a component is under debate and different stellar scenarios have been proposed, including the peculiar $s$-process activated in fast rotating massive stars at low metallicity [8] and a number of processes associated to the neutrino-driven winds in core-collapse supernovae [9].

The $s$-process nucleoysnthesis calculations in massive stars are affected by the nuclear uncertanties associated to neutron capture reaction rates. Most of Maxwellian-averaged cross-sections (MACS) of the isotopes in the mass region between $\mathrm{Fe}$ and $\mathrm{Sr}$ are lower than 150 mbarn. Their variation within their experimental uncertainties causes a propagation effect over heavier $s$-process abundances. In order to provide reliable weak $s$-process predictions all these neutron-capture cross sections should be determinated with the highest possible experimental accuracy (less than 10\%) at stellar conditions where the $s$-process is activated in massive stars, i.e. mainly in the convective He-burning core at $\sim 30 \mathrm{keV}$ and in the convective C-burning shell at $\sim 90 \mathrm{keV}$ (see e.g., [2]). Moreover, the weak $s$ predictions are also affected by the uncertainties of the neutron capture cross sections of the light neutron poisons like ${ }^{23} \mathrm{Na}$ [10]. The situation becomes more puzzling because the stellar rates may be not constrained equally well than the neutron capture cross sections [11]. The reason is that only the capture of the ground state is measured in the laboratory while the captures of excitated states can contribute considerably at typical $s$-process temperatures. Rauscher et al. [11] have proposed the ground-state contribution $(X)$ to the stellar reaction rate as a factor to be taken into account in the planning of future experiments. Low values of $X$ indicate that it is impossible to determine the stellar reaction rate directly from the experimental capture data, while the stellar enhancement factor could provide values close to the unity.

This paper shows some efforts to improve the quality and accuracy of the MACS used in stellar models. The MACS can be obtained by the time-of-flight (TOF) or the activation technique. We will describe some measurements already undertaken, and their implications in nucleosynthesis. Furthermore, new methods and challenging future experiments that can open a new window in the nuclear astrophysics field will be discussed.

\section{MACS by the Time-Of-Flight Technique: $n \_$TOF at CERN}

An example of important efforts in the measurement of MACS of relevant isotopes in the $s$-process is the n_TOF facility at CERN. It is based on the spallation process of the $20 \mathrm{GeV} / \mathrm{c}$ protons onto lead target. The features of the existing neutron beam (with a flight path of $185 \mathrm{~m}$ ) are: excellent TOF resolution, low intrinsic backgrounds, a wide energy range, from thermal to a few GeV and the high instantaneous neutron flux, resulting in a high signal-to-background ratio giving the possibility to measure the cross-section of high radioactive samples as those of the branching points [12]. We briefly summarize here one of them and its astrophysical implications, ${ }^{63} \mathrm{Ni}$, see details in [13]. New opportunities at $n \_$TOF at the new experimental area currently under construction will be discussed. 


\subsection{A representative example: ${ }^{63} \mathrm{Ni}(n, \gamma)$ cross-section}

${ }^{63} \mathrm{Ni}\left(T_{1 / 2}=101.2 \mathrm{yr}\right)$ is located in the path of the weak $s$-process and can became an important branching. During the He core burning the neutron density is low and the typical temperature $\mathrm{T}=0.3 \mathrm{GK}$ (26 $\mathrm{keV}$ ) leads to the $\beta$-decay of ${ }^{63} \mathrm{Ni}$ to ${ }^{63} \mathrm{Cu}$ in more than $90 \%$. But in the $\mathrm{C}$ shell burning the neutron density can be increased up to $10^{11-12} \mathrm{~cm}^{-3}$ as well as the temperature up to $1 \mathrm{GK}(90 \mathrm{keV})$. In these conditions the material is partly reprocessed and the ${ }^{63} \mathrm{Cu}$ can be bypassed producing ${ }^{64} \mathrm{Ni}$ and the subsequent chain, even if the half-life of the ${ }^{63} \mathrm{Ni}$ is reduced to a few years in the stellar environment. The following radiogenic decay of ${ }^{63} \mathrm{Ni}$ to ${ }^{63} \mathrm{Cu}$ will feed the final $s$-process yields of ${ }^{63} \mathrm{Cu}$.

The ${ }^{63} \mathrm{Ni}(n, \gamma)$ cross-section measurement was a challenge due to the production and handling of the high activity sample [13]. It was produced by irradiating a highly enriched ${ }^{62} \mathrm{Ni}$ sample in a thermal reactor. The irradiation took place more than 20 years ago, so the impurity of ${ }^{63} \mathrm{Cu}$ had to be chemically separated. The final sample consisted of $1156 \mathrm{mg}$ of $\mathrm{NiO}$ encapsulated powder. The ${ }^{63} \mathrm{Ni}(n, \gamma)$ cross-section was measured from thermal to $200 \mathrm{keV}$ resolving 12 resonances. The MACS was calculated from $k T=5$ to $100 \mathrm{keV}$. A factor of 2 higher than the theoretical predictions was found in all the energy range. The impact of the results on the weak $s$-process was investigated for massive stars, considering a $25 M_{\odot}$ star [14]. The abundance distribution prior to the supernova explosion showed an enhancement of $20 \%$ for ${ }^{64} \mathrm{Ni}$, a reduction of $15 \%$ for ${ }^{63} \mathrm{Cu}$, while ${ }^{65} \mathrm{Cu}$ synthesis was unchanged. Although the abundances in the $\mathrm{Ni}-\mathrm{Cu}-\mathrm{Zn}$ region are affected by the successive states of the stellar evolution, in particular by the explosive states, these results represent a fundamental improvement of the knowledge of the preexplosive $s$-process in massive stars.

\section{$2.21^{\text {st }}$ and forthcoming $2^{\text {nd }}$ Experimental Area: new opportunities}

Besides the measurement of ${ }^{63} \mathrm{Ni}(n, \gamma)$ cross-section, other quality results obtained at $\mathrm{n}_{-} \mathrm{TOF}$ in the Experimental Area 1 can be found in literature. We describe here some of the future measurements at $\mathrm{n} \_\mathrm{TOF}$, and the advantages of the $2^{\text {nd }}$ Experimental Area (EAR-2) currently under construction.

As we have discussed in Sect. 1 for meaningful abundance predictions the cross-sections of stable isotopes should be available with an accuracy of $10 \%$ or better, but uncertainties as low as $1 \%$ are desired for stable isotopes and in particular for a number of key isotopes to interpret the $s$-process signatures discovered in the presolar grains [15]. The goal of $1 \%$ uncertainty has been reached for only half of the cases between ${ }^{70} \mathrm{Ge}$ and ${ }^{204} \mathrm{~Pb}$. An effort in this direction will be carried out at $n$ TOF in the next experimental campaign starting in 2014 at CERN. The neutron-capture cross-section of all stable $\mathrm{Ge}$ isotopes will be measured in the energy range from thermal to $200 \mathrm{keV}$. Neutron induced reactions on Ge influence the abundance for heavier isotopes up to a mass number of $A=90$. It is expected to resolve resonances in the energy range up to $200 \mathrm{keV}$. It should be noticed that ${ }^{73} \mathrm{Ge}(n, \gamma)$ cross-section has never been measured before. More challenging neutron-capture cross-section measurements will be carried out at the n_TOF facility such as ${ }^{171} \mathrm{Tm}\left(T_{1 / 2}=1.92 \mathrm{yr}\right)$ and ${ }^{204} \mathrm{Tl}\left(T_{1 / 2}=3.78 \mathrm{yr}\right) .{ }^{171} \mathrm{Tm}$ is one of the branching points that is independent of stellar temperature and therefore it can be used to constrain $s$-process magnitudes as the neutron density. ${ }^{204} \mathrm{Tl}$ is interesting for the same reason and also because it determines the ${ }^{205} \mathrm{~Pb} /{ }^{205} \mathrm{Tl}$ clock for dating the early solar system. Both neutron-capture cross-sections have never been measured before. The samples will be produced by neutron irradiation of ${ }^{170} \mathrm{Er}$ and ${ }^{203} \mathrm{Tl}$, respectively, at the ILL nuclear reactor.

However, the greatest challenge for the $\mathrm{n}_{-}$TOF collaboration in the near future is the construction of a new experimental area (EAR-2) [16], started on June 2013. The new beam line will have a flight path of $20 \mathrm{~m}$ from the existing spallation target at $90^{\circ}$ with respect the proton beam and it will have an overground experimental area. The main advantage of the EAR-2 with respect to other facilities and in particular to the existing beam line EAR-1 is that the neutron flux will be increased by approximately 
25 times in all energy range. As a consequence neutron-induced reaction measurements can be carried out on smaller samples and on isotopes with lower cross-sections. These features are important for reducing the backgroung related to the activity of unstable isotopes thus allowing to optimize the signal-to-background ratio for neutron poisson in the s-process. Moreover, a reduction of the socalled $\gamma$-flash (a signal produced by the relativistic particles generated in the spallation process) is expected, because these particles seems to be emitted predominantly in the forward direction. In the future, it will be very useful to perform at EAR-2 some of the measurements discussed above.

\section{MACS by the Activation Technique: production of stellar spectra}

The concept of the activation technique is simple: a sample is irradiated by neutrons and the radioactivity of the radioisotope created is measured. If a stable isotope is produced, Accelerator Mass Spectroscopy can be used [17]. The sample can be irradiated by neutrons following any distribution, but in order to obtain the MACS, the cross-section obtained with this distribution must be corrected. The correction depends on the cross-section of the isotope. A problem exists if the cross-section is poorly known, as in the case of the major part of the branching points. In order to estimate the correction of the MACS, the cross-section must be known in the astrophysical energy range or it is necessary to rely on theoretical evaluations. In this section, we will briefly discuss the production of quasi maxwellian neutron spectrum (MNS) and a new method for MNS production [18]. We present here the astrophysical implications of the MACS of ${ }^{181} \mathrm{Ta}(n, \gamma)$ measured with this new method.

\subsection{Quasi Maxwellian Neutron Spectrum: Karlsruhe}

W. Pönitz [19] produced one of the first neutron beams following a quasi MNS at $k T=30 \mathrm{keV}$ by means of the ${ }^{7} \mathrm{Li}(p, n)^{7} \mathrm{Be}$ reaction near the threshold. He performed an accurate study of the ${ }^{197} \mathrm{Au}(n, \gamma)$ cross-section at $30 \mathrm{keV}$ due to its interest as a standard in astrophysical studies. The evaporation of $\mathrm{Li}(\mathrm{LiF})$ onto a $\mathrm{Cu}$ backing plate, the production of neutrons in a forward cone, the use of a water-cooling ring around the edge of the $\mathrm{Cu}$ backing plate which did not interfere with the neutron beam are the main features of the facility used for the measurements carried out at Karlsruhe. Few years later, Beer and Käppeler [20] improved this method, showing that a quasi MNS at $k T=23.4$ $\mathrm{keV}$ can be produced using a proton beam with energy $25 \mathrm{keV}$ above the threshold of the reaction ${ }^{7} \mathrm{Li}(p, n)^{7} \mathrm{Be}$. Finally, Ratynski and Käppeler [21] established $1.912 \mathrm{MeV}$ as the optimal proton energy beam for producing a quasi MNS at $k T=25.3 \mathrm{keV}$. As reported in [21], an accurate study of the ${ }^{197} \mathrm{Au}(n, \gamma)$ cross-section at $30 \mathrm{keV}$ was also carried out. The results of the MACS for Au were $(600 \pm 12)$ mbarn [19] and (582 \pm 9$)$ mbarn [21]. Even if the neutron spectra were different very similar values were obtained by both activations with quasi MNS, since each of them were corrected using the cross-section as a function of the energy, previously known.

In view of the results the question arise whether it is necessary to generate MNS at $k T=30 \mathrm{keV}$. The answer is that it is not needed, if the measured cross-section is previously known but it is certainly mandatory to use an exact MNS in case of poor knowledge of the cross-section (see Section 4). Moreover, the activation measurement is performed at one given $k T$ and the result is usually extrapolated to the relevant stellar temperatures relying on previous TOF measurements or on theoretical calculations. Unfortunately, for many isotopes TOF measurements are missing and the evaluations show important discrepancies. This fact was pointed out by G. Martín-Hernández et al. [22] showing the conflicting situation of a stable isotope, ${ }^{82} \mathrm{Se}$. Therefore, it is advantageous to generate MNS at different $k T$ s and within the same experimental setup, in order to measure the MACS at the $k T$ of interest and to reduce systematic uncertainties. 


\subsection{MACS by Proton Shaping: ${ }^{181} \mathrm{Ta}(n, \gamma)$, astrophysical implications}

Mastinu et al. [18] proposed a method for the generation of MNS with the aim of solving various issues of the existing methods: generation of MNS at $k T=30 \mathrm{keV}$, for the measurement of MACS by the activation technique for isotopes with unknown cross-sections such as those of several branching points and radioactive isotopes, low sensitivity to variations in the energy of the proton beam and generation of MNS at different $k T$ s with the same setup. The method is based on shaping the energy distribution of the proton beam close to the ${ }^{7} \mathrm{Li}(p, n)^{7} \mathrm{Be}$ reaction threshold. This can be done by means of a simple foil or a rotating foil made of different materials and thicknesses.

Recently, J. Praena et al. [23] have shown the measurement of the proton beam distribution as a validation of the proton beam shaping, and a comparison of the simulated neutron spectra with the experimental spectra at different angles reported in [24] and with the angle-integrated spectra of [21] as a validation of the neutron transport method. Although the simulations are reliable it is mandatory to experimentally validate the method. For this purpose an experiment was carried out at JRC-IRMM (Geel, Belgium) in the framework of the EUFRAT project. It consisted of a $3.663 \mathrm{MeV}$ proton beam passing through $75 \mu \mathrm{m} \mathrm{Al}$ foil acting as shaper. The proton beam hit a LiF target deposited onto a $\mathrm{Cu}$ backing. It was pulsed at $625 \mathrm{kHz}$, with $1.5 \mathrm{~ns}$ width and a flight path of $52 \mathrm{~cm}$ was chosen. The generated neutrons were measured at different angles by ${ }^{6} \mathrm{Li}$-glass detectors from $0^{\circ}$ to $80^{\circ}$ in steps of $10^{\circ}$. Figure 1 (left) shows experimental data at $0^{\circ}$ (red) compared with simulations (black). In this preliminary analysis the ${ }^{6} \mathrm{Li}(n, t)^{4} \mathrm{He}$ cross-section has been used as detector efficiency. There are still few corrections missing: detector efficiency, resolution function and background subtraction. Nevertheless, these results are a good indication of the experimental validation of our method.
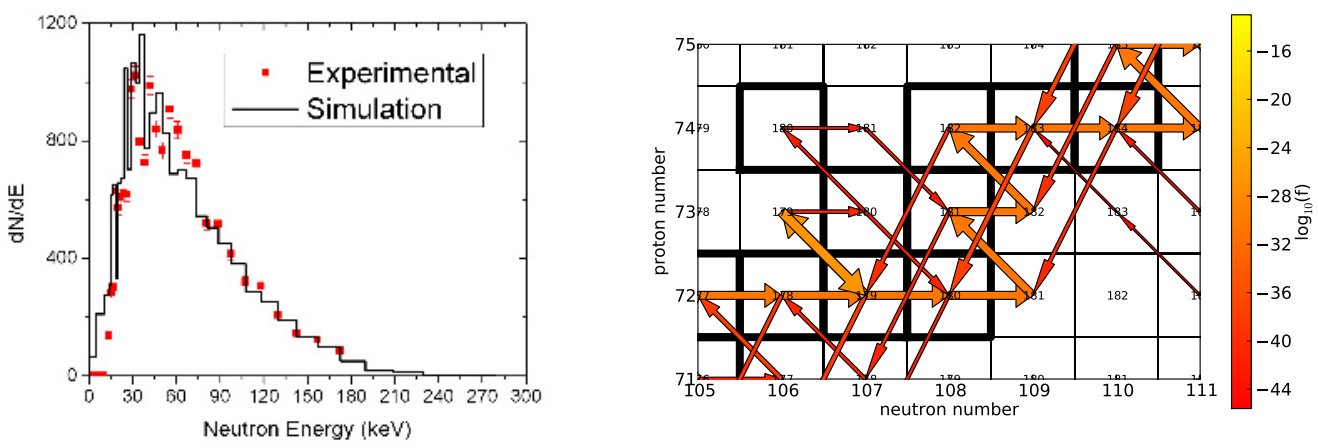

Figure 1. Left. Experimental neutron spectrum (arb. units) (red) at $0^{\circ}$ compared with simulations (black). Right. Neutron capture fluxes $\left(d Y_{i} / d t\right.$, where $\left.Y_{i}=X_{i} / A_{i}\right)$ in the Ta mass region $(Z=73)$ are presented for ${ }^{13} \mathrm{C}$-pocket conditions, with temperature of about $10^{8} \mathrm{~K}$, density of about $10^{2-3} \mathrm{~cm}^{-3}$, and neutron density of about $10^{7} \mathrm{~cm}^{-3}$. Thicker arrows and with more yellow colours indicate a larger flux relevance.

With the method described above, the MACS of the ${ }^{181} \mathrm{Ta}(n, \gamma)$ reaction at $k T=30 \mathrm{keV}$ has been measured at the Tandem $3 \mathrm{MV}$ at CNA, see details in [23]. A value equal to $(815 \pm 73)$ mbarn is reported using the MACS of the ${ }^{197} \mathrm{Au}(n, \gamma)$ reaction at $k T=30 \mathrm{keV}$ [21] as a reference. Most of the Tantalum $(Z=73)$ in the solar system is made of ${ }^{181} \mathrm{Ta}(99,988 \%)$, whereas a tiny fraction is made of ${ }^{180} \mathrm{Ta}$, that is the rarest stable isotope $\left(0.02\right.$ in meteoritic scale, where $\left.\mathrm{Si}=10^{6}\right)$. About $46.5 \%$ of the solar ${ }^{181} \mathrm{Ta}$ is reproduced by the $s$-process in low mass AGB stars [25]. Applying the residual method to the solar abundance distribution [26], the contribution to ${ }^{181} \mathrm{Ta}$ by the explosive $r$-process is about 
53.5\%. Such a prediction depends also on the ${ }^{181} \mathrm{Ta}(\mathrm{n}, \gamma)$ cross section used in stellar nucleosynthesis calculations. Indeed, because of the local equilibrium reached in $s$-process conditions in low mass stars, e.g. [27], a variation of ${ }^{181}$ Ta MACS will have no relevant impact on heavier species along the $s$-process path, but will inversely affect the final ${ }^{181}$ Ta yields.

In order to discuss the impact of the ${ }^{181} \mathrm{Ta}(\mathrm{n}, \gamma)$ MACS, we present the nucleosynthesis postprocessing calculations for a low mass AGB star with initial mass of $2 M_{\odot}$ and solar metallicity. The code PPN [28] is used for the simulations with a nuclear network of about 1100 species and 15000 reaction rates. Among relevant reactions involving light species, for the main neutron source ${ }^{13} \mathrm{C}(\alpha, n){ }^{16} \mathrm{O}$ the reaction rate is given by [29]. Neutron captures on stable species and for few relevant unstable species close to the valley of stability are taken from [30]. In other cases, REACLIB rates are used [31]. In particular, for the ${ }^{181}$ Ta MACS the default rate is given by [32]. Finally, electron-capture rates and $\beta$-decays in the Ta mass region are given by [33], or in case the stellar rate is not available (for instance, for species far from the valley of stability), the terrestrial rates are used.

In low-mass AGB stars, more than $90 \%$ of the total amount of neutrons are provided by the ${ }^{13} \mathrm{C}(\alpha, n){ }^{16} \mathrm{O}$ reaction, which is efficiently activated in a tiny zone called ${ }^{13} \mathrm{C}$-pocket and located within the $\mathrm{H}$ shell and the He shell during the radiative phase, between two following thermal pulses. The rest of the neutrons is provided by the partial activation of the ${ }^{22} \mathrm{Ne}(\alpha, n)^{25} \mathrm{Mg}$ at higher temperatures and higher neutron densities during the thermal pulses [34]. Figure 1 (right) shows the neutron capture conditions in the ${ }^{13} \mathrm{C}$-pocket where the neutron density is quite low, and a typical unbranched $s$-process pattern is obtained. On the other hand, the higher neutron density triggered by the ${ }^{22} \mathrm{Ne}(\alpha, n)^{25} \mathrm{Mg}$ activation during the thermal pulses (see figure 2 on the left), causes the open of different branchings, in particular for ${ }^{181} \mathrm{Hf}(Z=72)$, with decay time scale of about a day at stellar conditions [33]. This implies that during the thermal pulses, the $s$-process production of ${ }^{181} \mathrm{Ta}$ is more complex than with lower neutron densities, possibly affected also by the ${ }^{181} \mathrm{Hf}$ branching. Figure 2 (right) reports the final $s$-process abundances in the Tantalum region for a $2 M_{\odot}$ AGB star and solar metallicity, calculated using the new ${ }^{181}$ Ta MACS. The new MACS causes a decrease of ${ }^{181} \mathrm{Ta}$ yields by about $10 \%$, with other $s$-process abundances only marginally affected. Such effect is due to the new ${ }^{181}$ Ta MACS $10 \%$ higher compared to the recommended by [30].
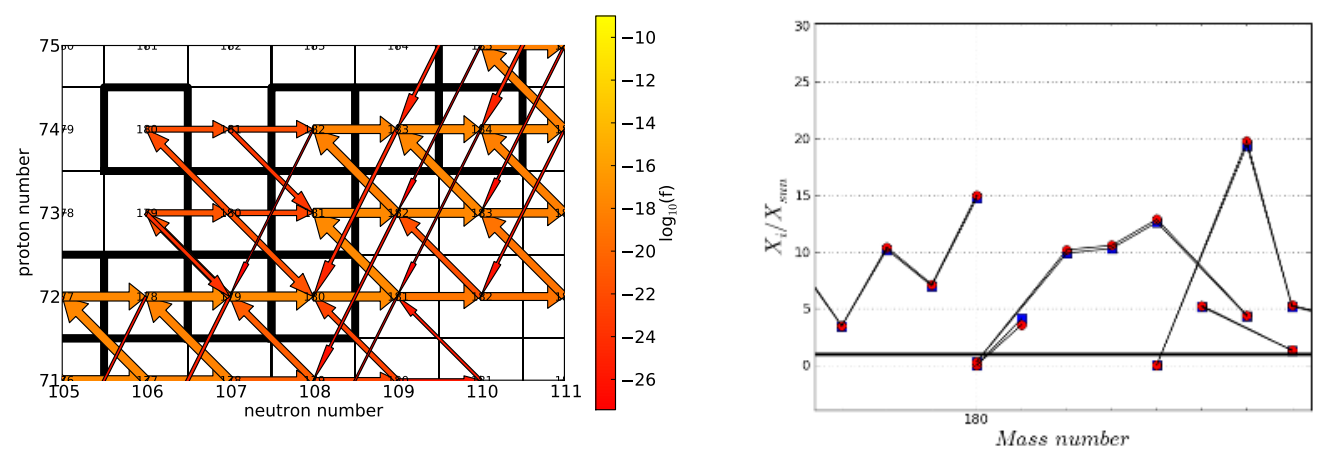

Figure 2. Left. Neutron capture fluxes at the bottom of the convective thermal pulse, with $\mathrm{T} \approx 2.6 \times 10^{8} \mathrm{~K}$, density of few $10^{3} \mathrm{~cm}^{-3}$, and neutron density of about $10^{10} \mathrm{n} \cdot \mathrm{cm}^{-3}$. Right. Production factors (abundance distribution normalized to solar) in the Ta region, using the default ${ }^{181}$ Ta MACS (blue squares), and the new cross section measurement reported in this work (red circles). The new abundance of ${ }^{181} \mathrm{Ta}$ decreases by about $10 \%$. 
The method described above allows the direct measurement of MACS at $30 \mathrm{keV}$ by activation of completely unknown cross-sections. Other improvements of the method with respect the previous ones are: a better control of the corrections, less sensitivity to potential fluctuations in the proton beam energy compared to quasi-MNS methods, and the production of MNS from $30 \mathrm{keV}$ to around $60 \mathrm{keV}$ in the same setup by means of the increment of the proton energy [35] or the enhancement of the target-to-sample distance [22].

\section{Future: In Situ Radioactive and Maxwellian Neutron Beams at SPES}

The SPES (Selective Production of Exotic Species) facility at LNL (INFN, Padova, Italy) currently under construction provides an unique opportunity to perform a new kind of MACS measurements of radioactive isotopes with completely unknown cross-section [36]. The aim of the SPES project [37] is to build an ISOL facility for the acceleration of radioactive beams (RIB) up to approximately $11 \mathrm{MeV} /$ nucleon for $A=130$. The high-intensity beams will open new possibilities in the study of nuclear astrophysics. In particular, isotopes around the neutron shell closures $(N=50,82$ and 126) are waiting points in the $r$-process chain, and they will be produced with enough intensity for halflife and mass measurements $\left({ }^{80} \mathrm{Zn},{ }^{81} \mathrm{Ga},{ }^{82} \mathrm{Ge},{ }^{83} \mathrm{As},{ }^{130} \mathrm{Cd},{ }^{132} \mathrm{Sn},{ }^{133} \mathrm{Sb} ..\right)$. Moreover, at SPES, it might be possible to deliver in situ a high-intensity RIB and a high-flux neutron beam, which can be of great advantage for measuring the MACS at $30 \mathrm{keV}$ of short-life isotopes never measured before. The high-flux neutron beam following a MNS at $30 \mathrm{keV}$ would be produced by means of the high intensity $5 \mathrm{MeV}$ proton beam of the RFQ accelerator of the TRASCO project at LNL. A possible setup is discussed in [18] and its status can be found in [35].

As an example, we describe here one of these possible future measurements, the MACS at $k T=30$ $\mathrm{keV}$ of the ${ }^{139} \mathrm{Ba}(n, \gamma)$ reaction. The first step would be the implantation of ${ }^{139} \mathrm{Ba}\left(T_{1 / 2}=83 \mathrm{~min}\right)$ onto a graphite substrate. The expected intensity of ${ }^{139} \mathrm{Ba}$ at SPES is $10^{10} \mathrm{~s}^{-1}$ and thereby around $6 \cdot 10^{13}$ atoms could be implanted. When saturation is reached (few hours), neutron irradiation with a MNS at $30 \mathrm{keV}$ begins while the RIB implantation continues. The expected neutron flux is $10^{11} \mathrm{n} /\left(\mathrm{s} \cdot \mathrm{cm}^{2}\right)$ considering the RFQ accelerator [18]. The in situ neutron and RIB irradiation should last 7 days. Although there is no experimental data we consider 0.1 barn as the MACS at $30 \mathrm{keV}$ of ${ }^{139} \mathrm{Ba}(n, \gamma)$ based on evaluations. Then, $6 \cdot 10^{5}{ }^{140} \mathrm{Ba}\left(T_{1 / 2}=12.7 \mathrm{~d}\right)$ nuclei should be created. A cooling time of 2 days is a good compromise between the elimination of background and the half-life of ${ }^{140} \mathrm{Ba}$. Finally, considering a detection efficiency of 0.09 , the $\gamma$-ray line of $537 \mathrm{keV}(I=0.25)$ and a measuring time of 11 days, $5 \cdot 10^{3}$ counts could be recorded.

Certainly, this kind of measurements are a great challenge and many issues must be solved before they can be succesfully performed. Still, it should be remarked that any MACS measurement of radioactive isotopes can provide a very important information, in particular, in view of the recent results in stellar evolution, which suggest a superpulse at the beginning of the He burning phase in low metallicity stars. The very high neutron densities could exceed the predicted ones for such superpulses implying that the $s$-process path would be shifted by a few mass units in the region of the $\beta$-unstable nuclei, thus hugely increasing the number of unstable isotopes in the $s$-process [16].

\section{Acknowledgments}

J. Praena is grateful to N. Colonna for the advices that improved the quality of the talk. Authors thank A. Krasa and F.-J. Hambsch for their invaluable help at JRC-IRMM. This work was supported by EUFRAT project (EU), spanish contracts FPA2009-08848, FPA2011-28770-C03-02, P07-FQM02894, and the funding agencies of the participating institutes. M. Pignatari acknowledges the support 
to NuGrid from NSF grants PHY 02-16783 and PHY 09-22648 (Joint Institute for Nuclear Astrophysics, JINA) and EU MIRG-CT-2006-046520, from the Ambizione grant of the SNSF and the SNF grant 200020_132816 (Switzerland) and Eurogenesis (MASCHE).

\section{References}

[1] E.M. Burbidge, G.R. Burbidge, W.A. Fowler, F. Hoyle, Rev. Mod. Phys. 29, 547 (1957).

[2] M. Pignatari, R. Gallino, M. Heil, M. Wiescher, F. Käppeler et al. , ApJ 710, 1557 (2010).

[3] F. Herwig, Annu. Rev. Astron. Astrophys, 43, 435 (2005).

[4] F.-K. Thielemann, A. Arcones et al., Prog. in Part. and Nuc. Phys. 66 2, 346 (2011).

[5] M. Arnould and S. Goriely, Phys. Rep., 384, 1 (2003).

[6] C. Travaglio, F. K. Röpke, R. Gallino and W. Hillebrandt, ApJ 739, 93 (2011).

[7] C. Travaglio, R. Gallino, E. Arnone, J. Cowan, F. Jordan, C. Sneeden, ApJ 601, 864 (2004).

[8] M. Pignatari, R. Gallino, G. Meynet, R. Hirschi, F. Herwig, M. Wiescher, ApJ 687, L95 (2008).

[9] C. Fröhlich, P. Hauser, M. Liebendörfer et al., ApJ 637415 (2006).

[10] M. Heil, F. Käppeler, E. Uberseder, et al. , Progr. Part. Nucl. Phys. 59, 174 (2007).

[11] T. Rauscher, P. Mohr, I. Dillmann, R. Plag, ApJ 738, 143 (2011).

[12] C. Guerrero, A. Tsinganis et al., (n_TOF Collaboration), Eur. Phys. J. A 49, 27 (2013).

[13] C. Lederer, C.Massimi et al., (n_TOF Collaboration), Phys. Rev. Let. 110, 022501 (2013).

[14] M. Pignatari, F. Herwig et al., ArXiv e-print, 2013arXiv1307.6961P (2013).

[15] M. Pignatari, E. Zinner, M. G. Bertolli et al., The Astro. J. Letters, 771, L7 (2013).

[16] E. Chiaveri et al., (n_TOF Collaboration). CERN-INTC-2011-032 ; INTC-O-013 (2011).

[17] A. Wallner, L. Coquard, I. Dillmann et al., J. Phys. G: Nucl. Part. Phys. 35014018 (2008).

[18] P.F. Mastinu, G. Martín-Hernández and J. Praena. NIM A 601, 333 (2009).

[19] W. Pönitz, Journal of Nuclear Energy Parts A/B, 20825 (1966), Pergamon Press Ltd.

[20] H. Beer and F. Käppeler. Phys. Rev. C 21, 534-544 (1980).

[21] W. Ratynski and F. Käppeler. Phys. Rev. C 37, 595-604 (1988).

[22] G. Martín-Hernández, P.F. Mastinu, J. Praena et al., App. Rad. and Isot. 70 8, 1583 (2012).

[23] J. Praena, P.F. Mastinu, M. Pignatari et al. NIM A, 727, 1 (2013).

[24] C. Lederer, F. Käppeler, M. Mosconi, R. Nolte et al. Phys. Rev. C 85 5, 055809 (2012).

[25] S. Bisterzo, R. Gallino, O. Straniero et al., ArXiv e-prints, arXiv:1108.0500 (2011).

[26] C. Arlandini, F. Käppeler, K. Wisshak et al., ApJ 525, 886 (1999).

[27] D.D. Clayton, Principles of stellar evolution and nucleosynthesis, NY, McGraw-Hill, 1968.

[28] F. Herwig, S. Diehl, C. Fryer et al., in Nuclei in the Cosmos (NIC X) arXiv:0811.4653 (2008).

[29] M. Heil, F. Käppeler, E. Uberseder, Phys. Rev. C 77, 015808 (2008).

[30] I. Dillmann, R. Plag, M. Heil et al., ArXiv e-prints, arXiv:0806.2016 (2008).

[31] T. Rauscher and F.-K. Thielemann, ADNDT 75, 1 (2000).

[32] K. Wisshak, F. Voss, F. Käppeler and G. Reffo, Phys. Rev. C 42, 1731 (1990).

[33] M. Aikawa, M. Arnould et al., A\&A 441, 1195 (2005).

[34] R. Gallino, C. Arlandini et al., ApJ 497, 388 (1998).

[35] P.F. Mastinu, J. Praena, G. Martín-Hernández et al., Physics Procedia 26, 261 (2012).

[36] J. Praena, P.F. Mastinu and G. Martín-Hernández, Pub. Astro. Soc. Australia, 36, 225 (2009).

[37] G. Prete et al., Journal of Physics: Conference Series 168, 012022 (2009). 